References

1 meNESTY, J., and DUHAMEL, P.: 'A fast exact least mean square adaptive algorithm', IEEE Trans., 1992, SP-40, (12), pp. 2904 2920

2 MOU, Z.J., and DUHAMEL, P.: 'Fast FIR filtering: Algorithms and implementations', Signal Process., 1987, 13, (4), pp. 377-384

3 HANSLER, E.: 'The hands-free telephone problem'. Proc. ISCAS San Diego, California, 1992, pp. 1914-1917

\section{New class of polyphase sequences with two- valued auto- and crosscorrelation functions}

P.Z. Fan, M. Darnell and B. Honary

Indexing term: Binary sequences

A new class of polyphase sequences with two-valued periodic auto- and crosscorrelation properties is proposed. It is proved that, for a given prime length $L>3$, the out-of-phase ACFs and CCFs of the sequences are constant and equal to $\sqrt{ } L$. It is also shown that sequences of the same length are mutually orthogonal and the correlation values asymptotically reach the Sarwate bound.

Introduction: Sets of sequences with good correlation properties have found application in radar, ranging and tracking, spreadspectrum communications, multiple-access communications, and system identification. For the correlation method of system identification, it is especially desirable that the out-of-phase autocorrelation functions (ACFs) of a sequence be constant. For multipleaccess communication purposes it is also desirable that the pairwise crosscorrelation functions (CCFs) between sequences assigned to various users be small, and preferably constant. There are many well-known classes of sequences having two-valued ACFs and small CCFs. For example, maximal length sequences, quadratic residue sequences, Frank sequences, Chu sequences, Lüke sequences, etc. [1-4]. In this Letter, a new class of polyphase sequences with near-optimal two-valued ACFs and CCFs is proposed. It is proved that, for a given prime length $L>3$, the outof-phase ACFs and CCFs of the sequences are constant and equal to $\sqrt{ } L$. An interesting and useful property of these sequences is that all the sequences of the same length $L$ are mutually orthogonal. It is shown that the correlation values asymptotically reach the Sarwate bound.

Sequences with two-valued ACFs and CCFs: For any integers $r, n$ and prime $L>3$, where $0 \leq n, r<L$, the new class of polyphase sequences, $a^{(r)}=\left(a_{0}^{(r)}, a_{1}{ }^{(r)}, \ldots, a_{L-1}(r)\right)$, is defined as

$$
a_{n}^{(r)}=\alpha^{n(n+1)(n+2) / 6+r n} \quad \alpha=e^{i 2 \pi v / L}
$$

where $\alpha$ is a primitive $L$ th root of unity and $v$ is any integer relatively prime to $L$. Obviously there exist $L$ sequences each of length $L$ for any prime $L>3$.

We now show that the above sequences have the following $\mathrm{ACF} / \mathrm{CCF}$ properties:

$$
\begin{aligned}
& \left|R_{r, \tau}(\tau)\right|= \begin{cases}L & \tau=0 \\
\sqrt{L} & \tau \neq 0\end{cases} \\
& \left|R_{r, s}(\tau)\right|= \begin{cases}0 & \tau=0, r \neq s \\
\sqrt{L} & \tau \neq 0\end{cases}
\end{aligned}
$$

The squared absolute value of the periodic CCF $R_{r, s}(\tau)$ between sequence $a^{(r)}$ and sequence $a(s)$ is defined as

$$
\left|R_{r, s}(\tau)\right|^{2}=\sum_{n=0}^{L-1} a_{n}^{(r)} a_{n+\tau}^{(s) *} \sum_{m=0}^{L-1} a_{m}^{(r) *} a_{m+\tau}^{(s)}
$$

Substituting eqn. 1 into eqn. 4, we obtain

$$
\begin{aligned}
& \left|R_{r, s}(\tau)\right|^{2} \\
& =\sum_{n=0}^{L-1} \alpha^{n(n+1)(n+2) / 6+r n-(n+\tau)(n+\tau+1)(n+\tau+2) / 6-s(n+\tau)} \\
& \sum_{m=0}^{L-1} \alpha^{-m(m+1)(m+2) / 6-r m+(m+\tau)(m+\tau+1)(m+\tau+2) / 6+s(m+\tau)} \\
& =\sum_{n=0}^{L-1} \alpha^{-\tau\left(2+6 n+3 n^{2}+3 \tau+3 n \tau+\tau^{2}\right) / 8-(s-r) n-s \tau} \\
& \sum_{m=0}^{L-1} \alpha^{\tau\left(2+6 m+3 m^{2}+3 \tau+3 m \tau+\tau^{2}\right) / 6-(s-r) m-s \tau} \\
& =\sum_{n=0}^{L-1} \sum_{m=0}^{L-1} \alpha^{\tau\left[6(m-n)+3\left(m^{2}-n^{2}\right)+3 \tau(m-n)\right] / 6+(s-r)(m-n)} \\
& =\sum_{n=0}^{L-1} \sum_{m=0}^{L-1} \alpha^{(m-n)[\tau(\tau+m+n+2) / 2+(s-r)]}
\end{aligned}
$$

When $\tau=\mathbf{0}$, it is obvious that

$$
\left|R_{r, s}(\tau)\right|^{2}=\sum_{n=0}^{L-1} \sum_{m=0}^{L-1} \alpha^{(m-n)(s-r)}= \begin{cases}L^{2} & \tau=0, s=r \\ 0 & \tau=0, s \neq r\end{cases}
$$

When $\tau \neq 0$, we introduce the following change of variables:

$$
n=m+l \quad l=0,1, \ldots, L-1
$$

$\left|R_{r, s}(\tau)\right|^{2}$ can then be rewritten as

$$
\begin{aligned}
\left|R_{r, s}(\tau)\right|^{2} & =\sum_{l=0}^{L-1} \sum_{m=0}^{L-1} \alpha^{-l[\tau(\tau+2 m+l+2) / 2+(s-r)]} \\
& =\sum_{l=0}^{L-1} \alpha^{-\tau l(\tau+l+2) / 2-l(s-r)} \sum_{m=0}^{L-1} \alpha^{-\tau l m} \\
& =L
\end{aligned}
$$

where

$$
\sum_{m=0}^{L-1} \alpha^{-\tau l m}= \begin{cases}0 & l \neq 0,(\tau, L)=1 \\ L & l=0\end{cases}
$$

It should be noted that for $\tau=0,1, \ldots, L-1, L$ must be a prime number in order to satisfy $(\tau, L)=1$.

We now consider the asymptotic performance of the sequences. Sarwate [5] has shown that for a family of $M$ uniform sequences, each of period $L$, the maximal magnitudes of the sidelobes $\boldsymbol{\Theta}_{a}, \boldsymbol{\theta}_{c}$ of auto- and crosscorrelation are lower bounded by

$$
\frac{\Theta_{c}^{2}}{L}+\frac{L-1}{L(M-1)} \frac{\Theta_{a}^{2}}{L} \geq 1
$$

For the proposed sequences, $M=L, \theta_{a}=\Theta_{c}=\sqrt{ } L$, the Sarwatebound yields

$$
\frac{(\sqrt{L})^{2}}{L}+\frac{L-1}{L(L-1)} \frac{(\sqrt{L})^{2}}{L}=1+\frac{1}{L} \geq 1
$$

which approaches the bound for large $L$. Therefore the above sequences are asymptotically optimal.

Example: As a simple example, let $L=7$; we obtain seven distinct sequences:

$$
\begin{aligned}
& a^{(0)}=\left(1, \quad \alpha, \quad \alpha^{4}, \quad \alpha^{3}, \quad \alpha^{6}, \quad 1, \quad 1\right) \\
& a^{(1)}=\left(1, \quad \alpha^{2}, \quad \alpha^{6}, \quad \alpha^{6}, \alpha^{3}, \quad \alpha^{5}, \alpha^{6}\right) \\
& a^{(2)}=\left(\begin{array}{lllllll}
1, & \alpha^{3}, & \alpha, & \alpha^{2}, & 1, & \alpha^{3}, & \alpha^{5}
\end{array}\right) \\
& a^{(3)}=\left(1, \quad \alpha^{4}, \quad \alpha^{3}, \quad \alpha^{5}, \quad \alpha^{4}, \quad \alpha, \quad \alpha^{4}\right) \\
& a^{(4)}=\left(\begin{array}{lllllll}
1, & \alpha^{5}, & \alpha^{5}, & \alpha, & \alpha, & \alpha^{6}, & \alpha^{3}
\end{array}\right)
\end{aligned}
$$

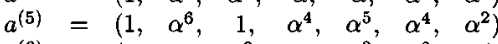

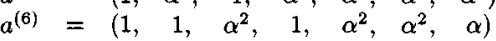

where $\alpha=e^{2 \pi / 7}$. Their auto- and crosscorrelation functions are given by

$$
\begin{aligned}
& \left|R_{r, r}(\tau)\right|=(7,2.6,2.6,2.6,2.6,2.6,2.6) \\
& \left|R_{r, s}(\tau)\right|=(0,2.6,2.6,2.6,2.6,2.6,2.6)
\end{aligned}
$$

where $r=s$. 
C IEE 1994

Electronics Letters Online No: 19940738

29 April 1994

PZ. Fan and M. Darnell (Department of Electronic Engineering. University of Hull, HU6 $7 R X$, United Kingdom)

B. Honary (Department of Engineering, Lancaster University, Lancaster, LAI $4 Y W$, United Kingdom

References

1 EVERETT, D.: 'Periodic digital sequences with pseudonoise properties', GEC J., 1966, 33, pp. 115-126

2 FRANK, R.L., and ZADOFF, S.A.: 'Phase shift pulse codes with good periodic correlation properties', IEEE Trans., October 1962, IT-8, pp. 381-382

3 CHU, D.C.: 'Polyphase codes with good periodic correlation properties', IEEE Trans., July 1972, IT-18, pp. 531-533

4 LUKE, H.D.: 'Families of polyphase sequences with near-optimal two-valued auto- and crosscorrelation functions', Electron. Lett. $1992,28,(1)$, pp. 1-2

6 SARWATE, D.V.: 'Bounds on crosscorrelation and autocorrelation of sequences', IEEE Trans., 1979, IT-25, pp. 720-724

\section{New TCM codes for 4PSK-2PSK modulation}

P. Szulakiewicz and R. Kotrys

Indexing terms: Phase shift keying, Trellis codes

The authors present some new multi-dimensional (3-D, 4PSK2PSK) and $180^{\circ}$ rotationally invariant trellis codes that combined with the demodulator (which locks onto the 2PSK signal of the 3D signal set) allows robust operation at low signal to noise ratios. Examples of the codes are presented for 2, 4, 8, 16 and 32 states. (t) $5.44 \mathrm{~dB}$ (for 32 encoder states) compared to uncoded BPSK. Distance profiles of the codes are shown.

New TCM codes for 4PSK-2PSK modulation: The authors present some new multi-dimensional (3-D, 4PSK-2PSK) and $180^{\circ}$ rotationally invariant trellis codes that combined with the demodulator (which locks onto the 2PSK signal of the 3-D signal set) allows robust operation at low signal to noise ratios. Examples of the codes are presented for $2,4,8,16$ and 32 states. The codes achieve a coding gain of $1.76 \mathrm{~dB}$ (for two encoder states) to $5.44 \mathrm{~dB}$ (for 32 encoder states) compared to the uncoded BPSK. Distance profiles of the codes are shown.

Introduction: When QPSK signals are encoded with a linear, rate $1 / 2$, convolutional code, it is only possible to achieve code invariance under $180^{\circ}$ signal rotation with certain codes, whereas with other codes no such invariance exists [1]. The lack of invariance for $90^{\circ}$ signal rotations requires that the QPSK signals be demodulated with the correct phase before Viterbi decoding. New nonlinear $90^{\circ}$ rotation invariant coded QPSK schemes were found in [1].

Another approach to the above problem is considered in this Letter. We consider linear TCM schemes with a 3-D constellation for sending one information bit per signalling interval. Each signal element is transmitted in the two consecutive signalling intervals as a pair of 2PSK and 4PSK symbols (Fig. 1a). This technique has been used previously with 4PSK-8PSK [2]

For coberent detection we have to extract the carrier phase from the received signal. In our case, for 2PSK-4PSK modulation we have the option of performing synchronisation with only two phase states (on the 2PSK signal set). Thus only $180^{\circ}$ synchronisation is required, increasing the robustness of the demodulator to noise. When 2PSK-4PSK signals are encoded, it is possible to easily achieve code invariance under $180^{\circ}$ signal rotation. Such codes were found for $2,4,8,16$ and 32 encoder states.

3-D signal configuration and its partitioning: Each signal element in the 3-D signal space is created by 2PSK and 4PSK signals transmitted in two consecutive signalling intervals. Fig. $1 a$ displays the
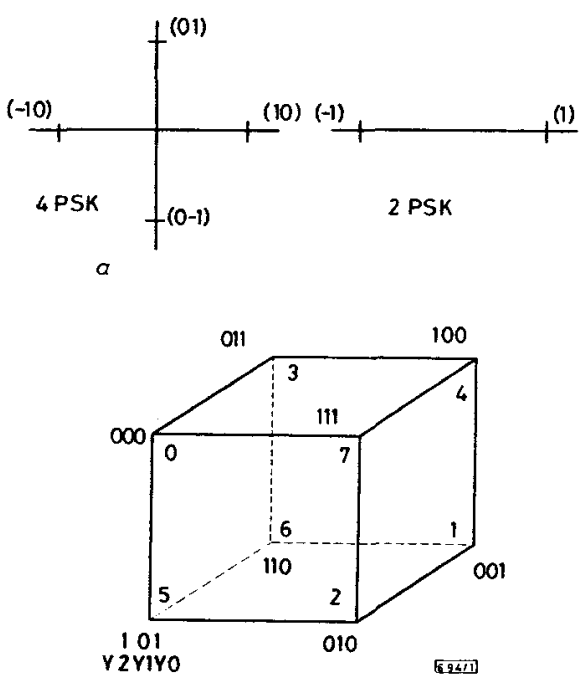

Fig. $12 P S K$ and 4PSK signal configuration, and 3-D signal space mapping for $180^{\circ}$ rotationally invariant TCM codes

a 2PSK and 4PSK signal configuration b 3-D signal space mapping

2PSK and 4PSK signal configurations. The average signal energy is one. Each point in the 3-D signal configuration is created by a pair of 2PSK and 4PSK signals. We assign binary numbers $Y_{2} Y_{1} Y_{0}$ to the signal elements as shown in Fig. $1 b$. We notice that a $180^{\circ}$ signal rotation always gives another signal with unchanged bits $Y_{2} Y_{1}$ and complementary bit $Y_{0}$. The minimum squared Euclidean distance within the $3-\mathrm{D}$ signal set $\{0,1,2,3,4,5,6,7\}$ is 2 , within the two subsets $\{0,2,4,6\}$ and $\{1,3,5,7\}$ the distance is 4 , and within subsets $\{0,4\},\{2,6\},\{1,5\},\{3,7\}$ the distance is 6 .

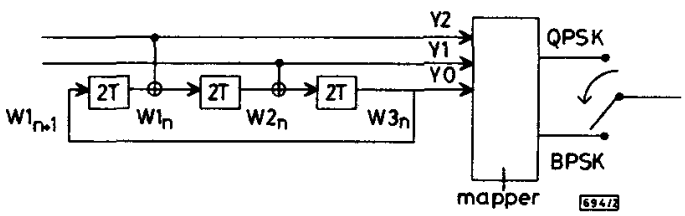

Fig. 2 Eight state encoder

Code design example: The design procedure follows the steps described in [3]. After set partitioning and state transition diagram definition the signal elements are assigned to the encoder state transitions. The assignment is performed such that the three Ungerboeck rules [4] are applied and rule A1 [3] is satisfied. To describe the code we use the parity check equation as given in [4] The encoder in Fig. 2 has (in octal notation) $h^{2}=04, h^{1}=02$ and $h^{0}=11$. Because $H^{0}(D)=D^{3} \oplus 1$ and $H^{0}(1)=0$, the code is $180^{\circ}$ invariant [7]. Distance $d^{2}{ }_{\text {min }}$ for the code of Fig. 2 is 10, hence the asymptotic coding gain of the code compared to uncoded BPSK is $10 \log (10 / 4)=3.98 \mathrm{~dB}$. Because $Y_{2} Y_{1}$ do not change on phase rotation, a differential encoder is not necessary.

Table 1: Code examples

\begin{tabular}{|c|c|rrr|r|r|c|}
\hline$v$ & $k$ & \multicolumn{1}{|c|}{$h^{2}$} & $h^{1}$ & $h^{0}$ & $d_{\text {free }}^{2}$ & $N_{\text {free }}$ & $\gamma$ \\
\hline & & & & & & & $\mathrm{dB}$ \\
\hline 2 & 2 & - & 2 & 3 & 6 & 2 & 1.76 \\
\hline 3 & 2 & 04 & 02 & 11 & 10 & 1 & 3.98 \\
\hline 4 & 2 & 14 & 02 & 33 & 12 & 2 & 4.77 \\
\hline 5 & 2 & 04 & 12 & 71 & 14 & 3 & 5.44 \\
\hline
\end{tabular}

Notation as in [7] 